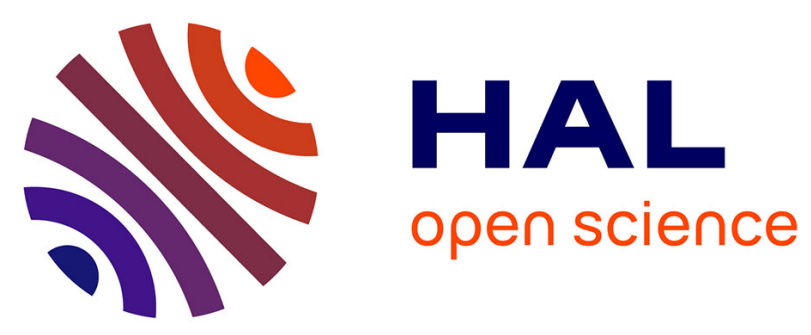

\title{
Genetic diversity of Echinococcus granulosus sensu stricto infecting humans in western Algeria
}

Daouia Moussa, Kheira Senouci, Nori Midoun, Mohamed Lacheheb, Benali

Tabeti, Noureddine Benmaarouf, Christophe Hennequin

\section{- To cite this version:}

Daouia Moussa, Kheira Senouci, Nori Midoun, Mohamed Lacheheb, Benali Tabeti, et al.. Genetic diversity of Echinococcus granulosus sensu stricto infecting humans in western Algeria. Parasitology Research, 2021, 120 (9), pp.3195-3202. 10.1007/s00436-021-07223-7 . hal-03365912

\section{HAL Id: hal-03365912 \\ https://hal.sorbonne-universite.fr/hal-03365912}

Submitted on 5 Oct 2021

HAL is a multi-disciplinary open access archive for the deposit and dissemination of scientific research documents, whether they are published or not. The documents may come from teaching and research institutions in France or abroad, or from public or private research centers.
L'archive ouverte pluridisciplinaire HAL, est destinée au dépôt et à la diffusion de documents scientifiques de niveau recherche, publiés ou non, émanant des établissements d'enseignement et de recherche français ou étrangers, des laboratoires publics ou privés. 
1 Genetic diversity of Echinococcus granulosus sensu stricto infecting humans in western

2 Algeria

3

4 Daouia Moussa $^{1}$, Kheira Senouci ${ }^{1}$, Nori Midoun ${ }^{2}$, Mohamed Lacheheb $^{3}$, Benali Tabeti ${ }^{4}$,

$5 \quad$ Noureddine Benmaarouf ${ }^{4}$, Christophe Hennequin 5 .

6

7 1. Natural and Life Sciences Faculty, Department of Biology, University of Oran 1 Ahmed 8 Ben Bella, Oran, Algeria.

9 2. Department of Epidemiology and Preventive Medicine, University Hospital of Oran 10 (EHU), Oran, Algeria.

11 3. Department of Thoracic Surgery, University Hospital of Oran (EHU), Oran, Algeria.

12 4. Department of Hepatobiliary and Liver Transplant, University Hospital of Oran (EHU),

13 Oran, Algeria.

14 5. Sorbonne Université, Inserm, Centre de Recherche Saint-Antoine, CRSA, AP-HP, Hôpital

15 Saint-Antoine, Service de Parasitologie-Mycologie, F-75012 Paris, France

16

17

18 Corresponding author:

19 C. Hennequin, Service de Parasitologie-Mycologie, Hôpital St Antoine, 34 rue Crozatier,

2075012 Paris, France

$21 \quad$ Phone + 33149283412

$22 \quad$ Fax +33149282251

23 Email: christophe.hennequin-sat@aphp.fr 


\section{Abstract:}

Human cystic echinococcosis is a zoonosis due to the flat worm Echinococcus granulosus sensu lato. The disease remains a major public health problem in Northern Africa. Molecular typing enables a better understanding of the parasite circulation from animals to humans. In this study we investigated the genotypic diversity of 46 Echinococcus granulosus isolates collected from humans in the western part of Algeria by the mean of partial sequences of 4 mitochondrial loci, namely cox $1 \mathrm{a}, \operatorname{cox} 1 \mathrm{~b}, \mathrm{nd} 3$ and atp6. Nucleotide polymorphism range from $0.6 \%$ (nd3) to $2.7 \%$ (cox1a). Eight alleles had not been previously reported. Multilocus analysis showed that all the isolates were from the Echinococcus granulosus sensu stricto (G1 genotype). Nineteen different haplotypes made of the concatenation of 4 sequenced loci were observed, the most common type clustering 13 isolates (36.1\%). Twelve of these haplotypes had never been described previously and fifteen $(41.7 \%)$ haplotypes were represented by only one isolate. Using sequences from this study and others retrieved from the Genbank database, any clustering either according to the geographic origin within Algeria or according to the human or animal origin of the isolates could be demonstrated supporting that genotype G1 population genetics has been shaped by intensive animal breeding. 40 1 2 3 44

Keywords: Echinococcus granulosus; cox1; nd3; atp6; mitochondrion; genetic diversity; Algeria. 7 48 
49 The authors declare that no funding was obtained for this study

50 The authors declare to have nothing to disclose

51 All the data and remaining materials are available to the scientific community

52 DM collected the samples, performed all the experiments and writes a first draft of the paper.

53 KS supervise the biological diagnosis and review the paper. NM, ML, NB and BT took care

54 of the patients and review the paper.

$55 \mathrm{CH}$ supervised the experiments, analyzed the data and writes the paper

56

57

58

59 


\section{Introduction}

Cystic echinococcosis (CE) is due to the development of the larval stage of the cestode Echinococcus granulosus sensu lato (s.l.). Human CE is a cosmopolitan zoonosis that represents a major public health problem in endemic countries where collective prophylaxis measures remain insufficient, notably in breeding areas such as North Africa (Dakkak 2010; Eckert et al. 2001). This is the case of Algeria where, despite improved educational hygiene and control over the slaughter of animals, the prevalence of CE remains high, with about 700 cases notified yearly (Dakkak 2010). This is due to the domestic life cycle of the parasite where dogs are the predominant definitive host of the parasite and the main source of contamination for humans, while intermediate hosts are represented by various species of ruminants. Previous reports from Algeria have shown that 16 to $42 \%$ of stray-dogs harbor E. granulosus s.l. in their intestine (Bentounsi et al. 2009), and that sheep, goat, cattle, horses, dromedaries, and wild boars can act as intermediate hosts, sheep being by far the most common (Bardonnet et al. 2003; Eckert et al. 2001; Kouidri et al. 2014; Laatamna et al. 2019; Maillard et al. 2007; Zait et al. 2016).

A high degree of genetic diversity has been documented in E. granulosus s.l. that partly correlates with intermediate host susceptibility (Alvarez Rojas et al. 2014; Romig et al. 2015). According to the current nomenclature, Echinococcus granulosus s.l. is now considered a species complex that includes Echinococcus granulosus sensu stricto (s.s.), Echinococcus equinus, Echinococcus ortleppi, Echinococcus felidis and Echinococcus canadensis (Alvarez Rojas et al. 2014; Romig et al. 2015). E. granulosus s.s. is the predominant species in many regions of the world, while E. canadensis, G6/G7 and, G8, G10 genotypes are dominant in eastern and north of Europe, respectively (Alvarez Rojas et al. 2014). E. granulosus s.s. includes 2 genotypes denoted G1 and G3, G2 being now considered a microvariant of G3 (Kinkar et al. 2017). The G1 genotype is predominant in all intermediate hosts, including Humans and is 
widely found in cattle in North Africa (Alvarez Rojas et al. 2014; Deplazes et al. 2017). G3, initially identified from water buffalo, can also infect other intermediate hosts including Humans (Romig et al. 2015).

Despite the importance of this public health problem in Algeria, epidemiological studies, and particularly molecular epidemiological studies, focused on E. granulosus s.l. from this country are still scarce. They conclude on the predominance of the E. granulosus s.s. G1 genotype as the main source of human infections (Bardonnet et al. 2003; Maillard et al. 2007; Zait et al. 2016). Some of these surveys may have limited conclusion notably due to length of the nucleotide sequence analyzed. However, such studies are important for different purposes. Even considering that the genotype is not strictly restricted to one intermediate host, the identification of the predominant species in a region would help health authorities to drive their prevention measures. Results of such studies can also give light on the circulation of the parasite between different regions, and to analyze the different hosts involved, including humans.

To increase our knowledge on the genetic diversity of E. granulosus s.l. circulating in Algeria, we initiated a genotyping study based on a 4 partial mitochondrial loci system focused on human isolates from the Western part of Algeria, where sheep breeding is mostly intensive. 


\section{Materials and methods}

\section{Biological samples}

104 Fluid from of 46 hydatid cysts (19 pulmonary and 27 hepatic) obtained after surgical resection

105 from 45 patients admitted at the Hospital of Oran (EHU) were available for the genotyping

106 study (Online Resource 1). Patients were from different localities mainly in the north-west of

107 Algeria (Figure S1). Fluids were first stored in $0.9 \%$ sodium chloride solution to avoid 108 protoscolex desiccation. They were then centrifuged at $50 \mathrm{xg}$ for $5 \mathrm{~min}$ and the pellet containing 109 protoscolex and/or the germinal layer were stored at $-20^{\circ} \mathrm{C}$ in $90 \%$ ethanol for further analysis.

\section{Molecular analysis}

\section{DNA extraction}

112 DNA was extracted from hydatid tissue samples (protoscolex and/or germinal layer) using a 113 mechanical disruption (MagnaLyser, Roche) followed by column-based extraction (QIAamp 114 DNA Blood kit, Qiagen) according to the manufacturer's instructions.

\section{Amplification and direct sequencing}

116 Four mitochondrial fragments were selected for genotyping (Figure S2). They target the

117 cytochrome c oxidase subunit 1 (2 fragments referred as coxla and cox $1 b$ ), the dehydrogenase

118 subunit 3 (nd3) and the ATPase subunit 6 (atp6). Briefly, amplification was performed with a 119 mixture containing $0.3 \mathrm{U}$ Taq polymerase (Taq Gold, Invitrogen), $1 \mu \mathrm{M}$ forward and reverse 120 primers and $5 \mu 1$ DNA in a final volume of $25 \mu 1$. Primers have been previously described and

121 their sequences are available in Online Resource 2 (Bowles et al. 1992; Lavikainen et al. 2006).

122 For all the targets, amplification was achieved according to the following thermal program: 10

123 min at $94{ }^{\circ} \mathrm{C}$, followed by 35 cycles of denaturation at $94^{\circ} \mathrm{C}$; hybridization at $50^{\circ} \mathrm{C}$ and 124 elongation at $72^{\circ} \mathrm{C}$, each of these steps lasted $30 \mathrm{sec}$; then a final extension at $72{ }^{\circ} \mathrm{C}$ during 10 125 min. Amplification was checked by running a 1\% agarose gel electrophoresis. 
126 PCR products were then purified (QIAquick PCR Purification kit) and subjected to direct 127 sequencing with the BigDye Terminator v3.1 reagent (Invitrogen), according to the 128 manufacturer's protocol and using the primers used for amplification. The corresponding

129 chromatograms were obtained using the $3500 \times 1$ Dx Genetic Analyzer automatic sequencer.

\section{Phylogenetic analysis}

131 The sense and anti-sense chromatograms were aligned and manually edited using the BioEdit 132 software version 7.2.6.Consensus sequences were then compared by the mean of the Blastn 133 program available on the National Center for Biotechnology Information website

134 (https://blast.ncbi.nlm.nih.gov/). Single locus multiple alignments were performed including 135 the reference mitochondrion genome (Genbank accession number AB786664) published by 136 Nakao et al.(Nakao et al. 2013)and using the MegaX (vs 10.1.8) software with the Clustal 137 algorithm.

138 Phylogenetic analysis was achieved using these sequences and sequences available in Genbank

139 and corresponding to Algerian E. granulosus s.s. isolates (Bardonnet et al. 2003; Kinkar et al. 140 2018; Laatamna et al. 2019; Maillard et al. 2007; Zait et al. 2016). We performed both an 141 analysis using the cox1blocus because a higher number of sequences are available in Genbank

142 with $100 \%$ query cover and another one using the concatenated sequences of the 4 loci further 143 in called haplotypes, obtained in our study. The phylogenetic relationship between sequences 144 identified in our series and sequences retrieved in Genbank was illustrated by the construction 145 of minimum spanning trees using an optimal eBurst algorithm (Francisco et al. 2009). Graphical 146 representation was done using the Phyloviz online program (http://www.phyloviz.net/).In this 147 graphical representation, isolates with the same allelic profile fall in the same circle, the size of 148 which is proportional to the number of isolates with that particular profile. 
151 The protocol has been approved by the Ethics Committee of the Hospital of Oran (ref. CA 152 O2/2020). Patients were informed during their hospital stay that data from their medical charts 153 could be used in a medical study that would be possibly published in a scientific journal. At the 154 beginning of the study, each specimen was given a code and all the experiments were performed 155 without knowledge of the patients' information, so that anonymization did not allow their 156 identification. 


\section{Results}

158 Amplification failed for the 4 genetic targets for two specimens. Positive amplification and sequencing were obtained for 37, 42, 43 and 43isolates for atp6, nd3, coxla, cox $1 b$, respectively making complete haplotype available for 36 isolates.

161 Single locus analysis revealed 8, 3, 10, 6allelesfor locus 1 to 4, respectively (Table 1). The level 162 of diversity with highest for coxla locus (2.7\%) and lowest for $n d 3$ locus (0.6\%). Eight of these

163 alleles have not been reported previously and have been deposited in GenBank (from MW417352 to MW417359 and MW446898-MW446899).

Phylogenetic analysis of concatenated sequences from our study and those found in GenBank showed that all our isolates were of E. granulosus s.s. G1 genotype (data not shown). Multilocus analysis on the 36 isolates with a complete haplotype allowed the differentiation of 19haplotypes named Hap01 to Hap19 (Table 2). Hap01 was the most common type isolates (36.1\%). Twelve of these haplotypes had never been reported previously and fifteen $(41.7 \%)$ haplotypes were represented by only one isolate. Notably $56 \%$ of the pulmonary isolates had

171 unique haplotype versus $20 \%$ for hepatic isolates.

172 The first phylogenetic tree was based on the coxlb locus taking the advantage of 92homolog

173 sequences in GenBank (Kinkar et al. 2018; Laatamna et al. 2019; Zait et al. 2016). It shows that $17436(83.7 \%)$ of our isolates harbor the predominant sequence (coxlb_01), similar to the reference 175 mitochondrial genome. Sequences from all the isolates but 2 were also direct satellites from 176 this central clade with links corresponding to a single nucleotide mutation. No segregation 177 according to the host nor to the geographic origin could be demonstrated (Fig1a, Fig 1b). When 178 using complete concatenated haplotypes using the sequences of four loci tested in our study, 179 Hap01 appeared as the founder, giving birth to closely related haplotypes either tested in this 180 study or retrieved in Genbank. However, Hap10, which originated indirectly from Hap01 (2 181 nucleotide mutations), forms a second cluster distantly with further diversification (Figure 2). 
182 All the sequences were obtained from cysts collected from humans and no apparent geographic

183 segregation trend was observed between these haplotypes.

184 
186 Because the life cycle of E. granulosus s.l. includes domestic animals, mainly dogs and

187 livestock, eradication of CE can be reached by control measures that could be set up from data

188 obtained in epidemiological surveys. There are several studies focused on the genetic

189 diversity of E. granulosus s.l. conducted in the Maghreb but those conducted in Algeria are

190 more rare (Bardonnet et al. 2003; Boufana et al. 2014; Kinkar et al. 2018; Laatamna et al.

191 2019; M'Rad et al. 2020; Oudni-M'rad et al. 2016; Zait et al. 2016).

192 In our study, we were able to genotype 44 E. granulosus s.l. isolates collected from humans

193 living in western Algeria. Previous studies have shown that whatever the country or the host

194 mitochondrial targets offer a high power of discrimination in regards to nuclear targets

195 (Laurimae et al. 2016; Lavikainen et al. 2006; Maillard et al. 2007). We used a multilocus

196 sequence typing scheme based on 4 fragments encoding mitochondrial genes with 1,626

197 nucleotides analyzed. This allowed the description of 19 haplotypes of which 12 had never been

198 reported, with 13 alleles newly described in Algeria of which 8 had not been previously

199 deposited in GenBank.

200 E. granulosus s.s. G1 genotype was the unique genotype identified for the all isolates tested in 201 this study. This is in accordance with previous studies conducted in Algeria that found a 202 frequency of 90.7 to $100 \%$ of this genotype in CE cases (Bardonnet et al. 2003; Laatamna et al. 2019; Zait et al. 2016). This also agrees with the worldwide predominance of E. granulosus s.s. that would cause $88.44 \%$ of the human CE cases (Alvarez Rojas et al. 2014). Surveys conducted in Tunisia and Morocco, two neighboring countries, also confirm that G1 genotype is 206 predominant as causing human CE in North Africa (M'Rad et al. 2005; Oudni-M'rad et al. 2016; Tahiri et al. 2019). However, while not detected in our study, it should be noted that both $E$. granulosus s.s. (G3) and E. canadensis (G6) have already been detected in Algeria (Maillard et al. 2007; Zait et al. 2016), the latter being mostly found in southern parts of Algeria where 
camelid breeding is common. These results are in accordance with the predominance of the G1

211 genotype in North Africa, warranting further studies to better understand the circulation of this species between the various hosts

213 Molecular epidemiology surveys having analyzed the genetic diversity of E. granulosus s.l. 214 diversity in Algeria remain rather rare. Zait et al. using a two-loci molecular system (coxlb and 215 nadl) identified twenty different haplotypes among the 70 isolates tested, mostly originating 216 from the Eastern part of Algeria, with a predominant one aggregating $71.6 \%$ of the isolates 217 being identical to the reference mitogenome (Zait et al. 2016). More recently, Laatamna et al, 218 used the full-length sequence of the coxl gene to demonstrate a particularly high genetic 219 diversity (73 different haplotypes among 125 isolates tested) in E. granulosus from livestock 220 animals from the Djelfa province(Laatamna et al. 2019). However, they also concluded on the 221 identity between the predominant haplotype and the reference mitogenome.

222 To enhance our knowledge on the genetic diversity of E. granulosus s.s. in Algeria, we compared our data with previously published sequences. Comparison was limited due to different selections of genetic targets and the length of sequences analyzed. Nevertheless, we were able to compared the haplotype of 92 isolates collected from different regions of Algeria using a part of the coxlgene (locus coxlb of our study). Indeed, partial or complete sequence of the coxl gene has been the most commonly used target for E. granulosus s.l. genotyping studies. Using this locus, we confirm the predominance of the coxla_Olallele, synonymous to the EG01 genotype, previously shown to cause most of human and animal CE cases in Algeria 230 (Laatamna et al. 2019; Zait et al. 2016). No segregation between isolates collected from humans 231 or animals was noted supporting the integration of both hosts in the life cycle of the parasite. 232 Similarly, Algerian isolates of the G1 genotype irrespective of their geographic origin, shared 233 a predominant haplotype or closely-related haplotypes, supporting the clonal expansion of the 234 parasite throughout the country without host barrier, with further diversification (satellite 
haplotypes with limited genetic variation). This is in accordance with the mode of reproduction

236 of the parasite that undergoes a sexual reproduction from a single worm thanks to a 237 hermaphrodite genital apparatus.

238 However, using the 4 loci scheme, we were able to detect a substantially higher level of genetic 239 diversity within the G1 genotype than using a single locus. All the haplotypes detected in our 240 study were genetically close to but different from the G1reference mitogenome available in 241 GenBank (AB786664) that has been isolated from a man in China (Nakao et al. 2013). As 242 previous studies conducted in other parts of Algeria, we showed that the Hap01 haplotype is 243 predominant in western Algeria. This synonymous nucleotide mutation, in a locus rarely 244 included in the typing method, has already been described in Italian, Australian, Tunisian and 245 Algerian isolates (Kinkar et al. 2019; Kinkar et al. 2018; Sgroi et al. 2019). The Hap01 246 haplotype belongs to a clonal complex that includes a low level of diversity with satellite 247 haplotypes (9 haplotypes diverge only by one single nucleotide mutation). However, the second most frequent haplotype Hap02, that derives from Hap01, seems to form a new complex that 249 gives birth to new closely related haplotypes. Whether this is due to the natural evolution of the 250 species or the introduction of either cattle or sheep, or dogs from other regions should be further 251 evaluated. The contamination of humans in other regions can also be hypothesized. 252 Interestingly, this haplotype has already been described from Kenchela, located in eastern 253 Algeria. Similarly, Hap04 was shared by two isolates from Boumerdes and Laghouat, 2 cities 254 distant from western Algeria. More recently, whole mitochondrial genome sequence using 255 primer walking has been proposed for E. granulosus s.l. typing and offers a better 256 discriminatory power and capability to analyze phylogenetic relationship). So, Kinkar et al. 257 showed that Algerian isolates of E. granulosus s.l. are split into different geographical clusters 258 that also include European and North African isolates (Kinkar et al. 2018). This and results 259 from other studies (Boufana et al. 2014; Hassan et al. 2017; Kinkar et al. 2018), notably showing 
260 the low fixation indices between human and animal isolates, strongly suggest that genotype G1

261 population genetics has been shaped by intensive animal trade.

262 While the results of the present study are based on a relatively low number of isolates, they 263 highlight the particularly high genetic diversity of E. granulosus s.s. in Algeria, with some 264 specific features. To improve our knowledge and better understand how the parasite spread into 265 this country and elsewhere, a consensus typing method should be defined allowing to aggregate 266 together the data from offering the opportunity to conduct robust phylogeography analysis. This 267 would be further applied to type both human and animal E. granulosus s.s. isolates 268 


\section{Acknowledgements}

271 This study was supported by the Parasitology laboratory of the University of Oran1 Ahmed

272 Ben Bella, the Department of Parasitology at the Hospital of Oran (EHU).

273 The authors would like to thank Chafika Tachema for referring the specimens.

274 The authors also thank the Joint Laboratory of Genetics and Molecular Biology and Sandra

275 Vellaissamy from the Parasitology Department of St Antoine Hospital for excellent technical 276 assistance.

277

\section{Conflict of interest}

279 The authors state that they have no conflict of interest.

280 


\section{References}

282 Alvarez Rojas, C. A., Romig, T., Lightowlers, M. W. (2014). Echinococcus granulosus sensu 283 lato genotypes infecting humans--review of current knowledge. Int J Parasitol 44 (1), 9-18. doi:10.1016/j.ijpara.2013.08.008

285

286

287

288

289

290

291

292

293

294

295

296

297

298

299

300

301

302

303

304

305

306

307

308

309

310

311

312

Bardonnet, K., Piarroux, R., Dia, L., Scheegans, F., Beurdeley, A., Godot V., Vuitton DA (2002) Combined eco-epidemiological and molecular biology approaches to assess Echinococcus granulosus transmission to human in Mauritania: occurrence of the camel strain and human cystic echinococcosis. Trans R Soc Trop Med Hyg 96:383-386

Bardonnet K, et al. (2003) Cystic echinococcosis in Algeria: cattle act as reservoirs of a sheep strain and may contribute to human contamination. Veterinary parasitology 116(1):3544 doi:10.1016/s0304-4017(03)00255-3

Alvarez Rojas CA, Romig T, Lightowlers MW (2014) Echinococcus granulosus sensu lato genotypes infecting humans--review of current knowledge. Int J Parasitol 44(1):9-18 doi:10.1016/j.ijpara.2013.08.008

Bardonnet K, et al. (2003) Cystic echinococcosis in Algeria: cattle act as reservoirs of a sheep strain and may contribute to human contamination. Vet Parasitol 116(1):35-44 doi:10.1016/s0304-4017(03)00255-3

Bentounsi B, Meradi S, Ayachi A, Cabaret J (2009) Cestodes of Untreated Large Stray Dog Populations in Algeria: A Reservoir for Herbivore and Human Parasitic Diseases. The Open Veterinary Science Journal 3:64-7

Boufana B, et al. (2014) Genetic variability and haplotypes of Echinococcus isolates from Tunisia. Trans R Soc Trop Med Hyg 108(11):706-14 doi:10.1093/trstmh/tru138

Bowles J, Blair D, McManus DP (1992) Genetic variants within the genus Echinococcus identified by mitochondrial DNA sequencing. Mol Biochem Parasitol 54(2):165-73 doi:10.1016/0166-6851(92)90109-w

Dakkak A (2010) Echinococcosis/hydatidosis: a severe threat in Mediterranean countries. Vet Parasitol 174(1-2):2-11 doi:10.1016/j.vetpar.2010.08.009

Deplazes P, et al. (2017) Global Distribution of Alveolar and Cystic Echinococcosis. Adv Parasitol 95:315-493 doi:10.1016/bs.apar.2016.11.001

Eckert J, Gemmell M, Meslin Fo-X, Pawlowski Z, Organization WH (2001) WHO/OIE manual on echinococcosis in humans and animals: a public health problem of global concern. 
Francisco AP, Bugalho M, Ramirez M, Carrico JA (2009) Global optimal eBURST analysis of multilocus typing data using a graphic matroid approach. BMC Bioinformatics 10:152 doi:10.1186/1471-2105-10-152

Hassan ZI, et al. (2017) Two haplotype clusters of Echinococcus granulosus sensu stricto in northern Iraq (Kurdistan region) support the hypothesis of a parasite cradle in the Middle East. Acta Trop 172:201-207 doi:10.1016/j.actatropica.2017.04.028

Kinkar L, et al. (2019) Long-read sequencing reveals a $4.4 \mathrm{~kb}$ tandem repeat region in the mitogenome of Echinococcus granulosus (sensu stricto) genotype G1. Parasit Vectors 12(1):238 doi:10.1186/s13071-019-3492-x

Kinkar L, et al. (2018) Global phylogeography and genetic diversity of the zoonotic tapeworm Echinococcus granulosus sensu stricto genotype G1. Int J Parasitol 48(910):729-742 doi:10.1016/j.ijpara.2018.03.006

Kouidri M, Benchaib Khoudja F, Boulkaboul A, Ammar Selles SM (2014) Comparative

Laurimae T, et al. (2016) Genetic diversity and phylogeography of highly zoonotic

M'Rad S, et al. (2005) Molecular evidence of ovine (G1) and camel (G6) strains of Echinococcus granulosus in Tunisia and putative role of cattle in human contamination. Vet Parasitol 129(3-4):267-72 doi:10.1016/j.vetpar.2005.02.006 
M'Rad S, et al. (2020) Microsatellite Investigations of Multiple Echinococcus Granulosus Sensu Stricto Cysts in Single Hosts Reveal Different Patterns of Infection Events between Livestock and Humans. Pathogens 9(6) doi:10.3390/pathogens9060444

Maillard S, et al. (2007) Taxonomic position and geographical distribution of the common sheep G1 and camel G6 strains of Echinococcus granulosus in three African countries. Parasitol Res 100(3):495-503 doi:10.1007/s00436-006-0286-9

Nakao M, et al. (2013) Mitochondrial phylogeny of the genus Echinococcus (Cestoda: Taeniidae) with emphasis on relationships among Echinococcus canadensis genotypes. Parasitology 140(13):1625-36 doi:10.1017/S0031182013000565

Oudni-M'rad M, et al. (2016) First molecular evidence of the simultaneous human infection with two species of Echinococcus granulosus sensu lato: Echinococcus granulosus sensu stricto and Echinococcus canadensis. Parasitol Res 115(3):1065-9 doi:10.1007/s00436-015-4836-x

Romig T, Ebi D, Wassermann M (2015) Taxonomy and molecular epidemiology of Echinococcus granulosus sensu lato. Vet Parasitol 213(3-4):76-84 doi:10.1016/j.vetpar.2015.07.035

Sgroi G, et al. (2019) Cystic echinococcosis in wild boars (Sus scrofa) from southern Italy: 


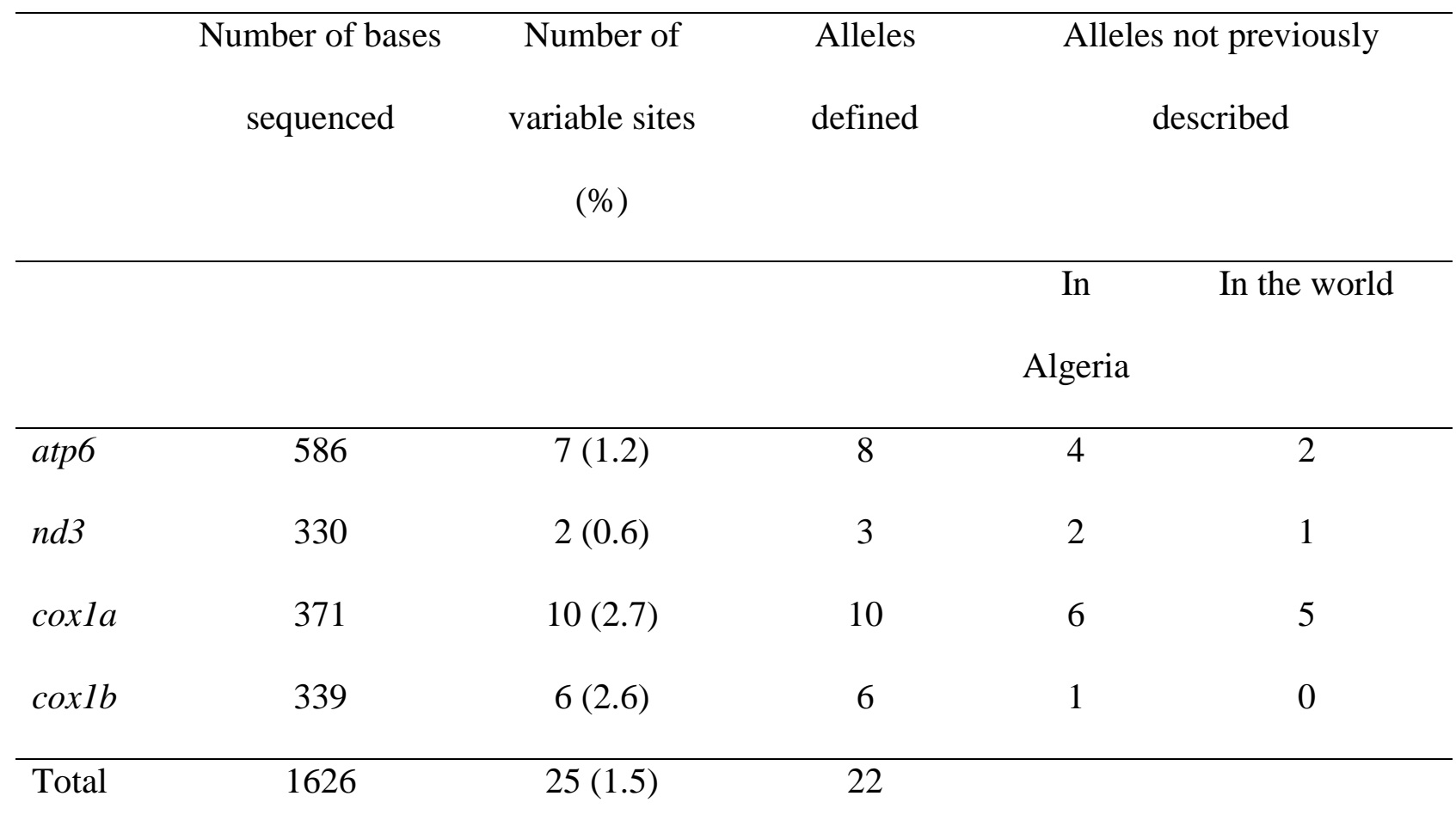

373

374

375 Table 1: Sequence variability of 4 mitochondria loci observed in this study 376

377 


\begin{tabular}{|c|c|c|c|c|c|c|c|c|c|c|c|c|c|c|c|c|}
\hline & \multicolumn{16}{|l|}{ atp6 } \\
\hline & 5802 & 5832 & 5841 & 5858 & 5861 & 5932 & 6060 & 6108 & 6126 & 6143 & 6145 & 6224 & 6286 & 6302 & 6329 & 6330 \\
\hline Reference & $\mathbf{T}$ & $\mathbf{G}$ & $\mathbf{C}$ & $\mathbf{C}$ & $\mathbf{T}$ & $\mathbf{T}$ & $\mathbf{C}$ & $\mathbf{T}$ & $\mathbf{A}$ & $\mathbf{T}$ & $\mathbf{G}$ & $\mathbf{T}$ & $\mathbf{A}$ & $\mathbf{C}$ & $\mathbf{A}$ & $\mathbf{T}$ \\
\hline apt6_01 & & & & & & & & & $\mathrm{G}$ & & & & & & & \\
\hline apt6_02 & & & $\mathrm{T}$ & & & & & & G & & & & & & & \\
\hline apt6_03 & & & & & $\mathrm{C}$ & & & & G & & & & & & & \\
\hline apt6_04 & & & & & & & & & G & & & & & $\mathrm{T}$ & & \\
\hline apt6_05 & & & $\mathrm{T}$ & $\mathrm{T}$ & & & & & G & & & & & & & \\
\hline apt6_06 & & & $\mathrm{T}$ & & & & & & G & & & & $\mathrm{T}$ & & & \\
\hline apt6_07 & & A & & & & & & & G & & & & & & & \\
\hline apt6_08 & $\mathrm{C}$ & & & & & & & & G & & & & & & & \\
\hline apt6_09* & & & & & & & $\mathrm{T}$ & & G & & & & & & & \\
\hline apt6_10* & & & & & & & & & G & & & & & & G & \\
\hline apt6_11* & & & $\mathrm{T}$ & & & & & & G & $\mathrm{C}$ & & & & & & \\
\hline apt6_12* & & & & & & $\mathrm{C}$ & & & G & & & & & & & \\
\hline \multirow[t]{3}{*}{ apt6_13* } & & & & & & & & $\mathrm{C}$ & G & & A & $\mathrm{C}$ & & & & $\mathrm{C}$ \\
\hline & $n d 3$ & & & & & & & & & & & & & & & \\
\hline & 8681 & 8726 & 8738 & 8820 & 8828 & 8842 & 8843 & & & & & & & & & \\
\hline Reference & $\mathbf{C}$ & $\mathbf{T}$ & $\mathbf{T}$ & $\mathbf{C}$ & $\mathbf{T}$ & $\mathbf{A}$ & $\mathbf{C}$ & & & & & & & & & \\
\hline \multicolumn{17}{|l|}{$n d 3 \_01$} \\
\hline nd3_02 & & & & $\mathrm{T}$ & & & & & & & & & & & & \\
\hline nd3_03 & & & & & & $\mathrm{T}$ & & & & & & & & & & \\
\hline$n d 3 \_04 *$ & $\mathrm{~T}$ & & & & & & & & & & & & & & & \\
\hline$n d 3 \_05 *$ & & & $\mathrm{C}$ & & & & & & & & & & & & & \\
\hline$n d 3 \_06 *$ & & $\mathrm{C}$ & & & C & & $\mathrm{T}$ & & & & & & & & & \\
\hline
\end{tabular}




\begin{tabular}{|c|c|c|c|c|c|c|c|c|c|c|c|c|c|c|c|}
\hline & \multicolumn{15}{|l|}{ coxla } \\
\hline & 9019 & 9038 & 9042 & 9052 & 9054 & 9072 & 9104 & 9132 & 9134 & 9137 & 9148 & 9169 & 9170 & 9207 & 9242 \\
\hline Reference & G & $\mathbf{A}$ & $\mathbf{C}$ & $\mathbf{T}$ & $\mathbf{A}$ & $\mathbf{G}$ & $\mathbf{C}$ & $\mathbf{A}$ & $\mathbf{A}$ & $\mathbf{A}$ & $\mathbf{T}$ & $\mathbf{T}$ & G & $\mathbf{A}$ & $\mathbf{C}$ \\
\hline \multicolumn{16}{|l|}{ coxla_01 } \\
\hline coxla_02 & & & & & & & & & & & & & & $\mathrm{G}$ & \\
\hline coxla_03 & & & & & & & & $\mathrm{G}$ & & & & & & & \\
\hline coxla_04 & & & & & & & & & & & & & A & & \\
\hline coxla_05 & & & & & & & & & & $\mathrm{G}$ & & & & & \\
\hline coxla_06 & & & $\mathrm{T}$ & & & & & & $\mathrm{G}$ & & & & & & \\
\hline coxla_07 & & & & & & & & & & & & $\mathrm{C}$ & & & \\
\hline coxla_08 & & & & & & & & & & & $\mathrm{C}$ & & & & \\
\hline coxla_09 & & & & & & & & & & & & & & & $\mathrm{T}$ \\
\hline coxla_10 & & & & & & & $\mathrm{T}$ & & & & & & & & \\
\hline coxla_11* & & G & & $\mathrm{C}$ & $\mathrm{G}$ & & & & & & & & & & \\
\hline coxla_12* & & & & & & A & & & & & & & & & \\
\hline \multirow[t]{3}{*}{ coxla_13* } & $\mathrm{A}$ & & & & & & & & & & & & & & \\
\hline & $\operatorname{cox} 1 b$ & & & & & & & & & & & & & & \\
\hline & 9822 & 9830 & 9853 & 9863 & 9870 & 9908 & 10054 & 10073 & & & & & & & \\
\hline Reference & $\mathbf{A}$ & $\mathbf{G}$ & $\mathbf{C}$ & $\mathbf{C}$ & $\mathbf{T}$ & $\mathbf{C}$ & $\mathbf{T}$ & $\mathbf{A}$ & & & & & & & \\
\hline coxlb_01 & & & & & & & & & & & & & & & \\
\hline coxlb_02 & & & $\mathrm{T}$ & & & & & & & & & & & & \\
\hline coxlb_03 & & & & $\mathrm{T}$ & & & & & & & & & & & \\
\hline coxlb_04 & & & & & & $\mathrm{T}$ & & G & & & & & & & \\
\hline cox1b_05 & & & & & $\mathrm{C}$ & & & & & & & & & & \\
\hline coxlb_06 & G & & & & & & & & & & & & & & \\
\hline coxlb_07* & & A & & $\mathrm{T}$ & & & $\mathrm{C}$ & & & & & & & & \\
\hline
\end{tabular}


384 Table 2: Polymorphic sites of the 4mitochondrial loci used in this study.

385 Polymorphism in comparison with the reference mitochondrial genome (genotype G1, GenBank accession number AB786664) are indicated in

\section{Bold}

387 *Denotes sequences retrieved in GenBank

388 


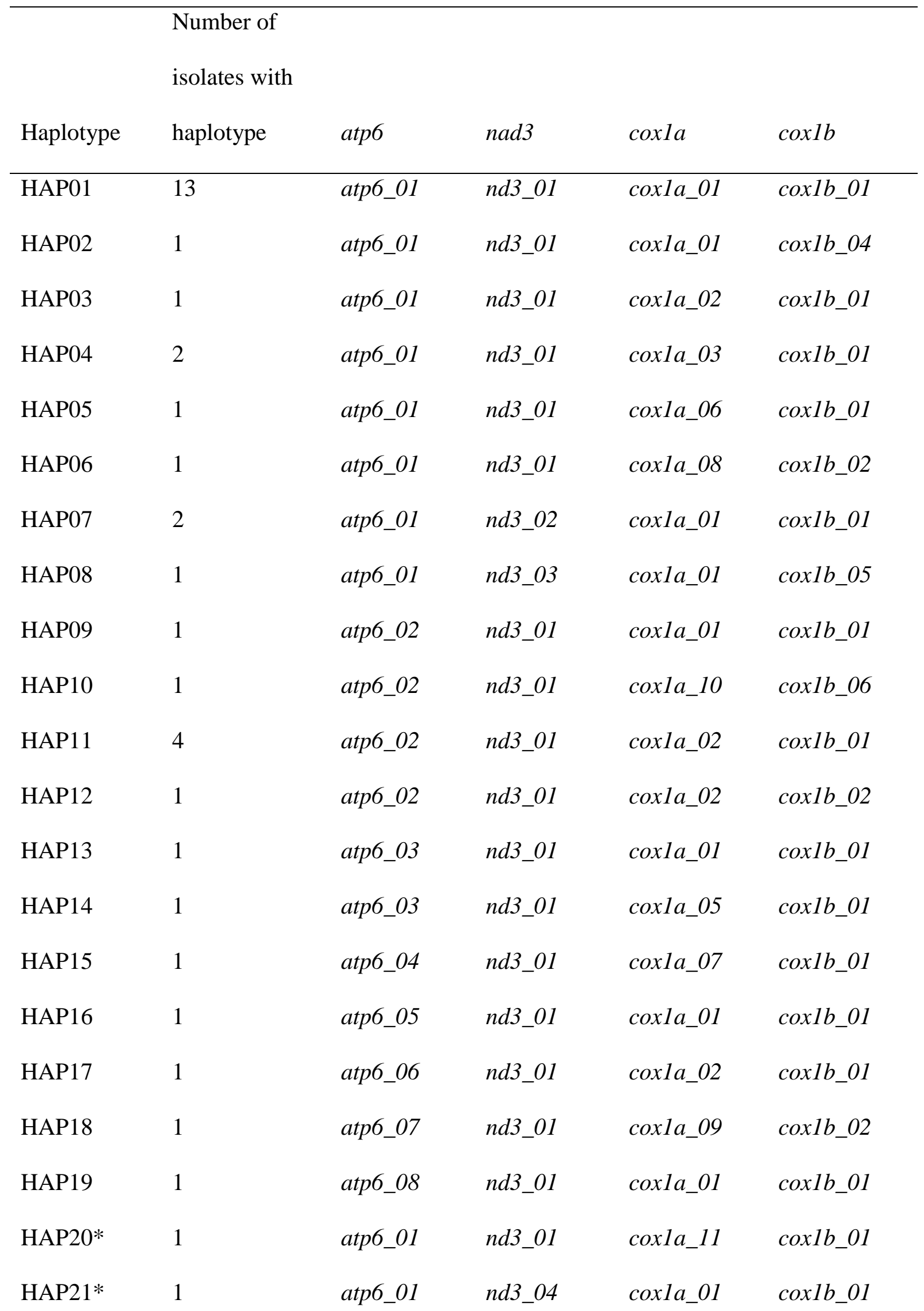




\begin{tabular}{lccccc} 
HAP22 & 1 & atp6_03 & nd3_05 & coxla_01 & coxlb_01 \\
HAP23* & 1 & atp6_09 & $n d 3 \_01$ & coxla_01 & coxlb_03 \\
HAP24* & 1 & atp6_10 & $n d 3 \_01$ & coxla_01 & coxlb_01 \\
HAP25* & 1 & atp6_11 & $n d 3 \_01$ & coxla_02 & coxlb_01 \\
HAP26* & 1 & atp6_12 & $n d 3 \_04$ & coxla_09 & coxlb_01 \\
HAP27* & 1 & atp6_01 & $n d 3 \_01$ & coxla_12 & coxlb_01 \\
HAP28* & 1 & atp6_13 & $n d 3 \_06$ & coxla_13 & coxlb_07 \\
\hline
\end{tabular}

391

392 Table 3: Allelic profiles of each haplotype

$393 *$ denotes haplotypes found in Genbank

394 
396 Fig. 1. Minimum spanning tree of the coxlb locus drawn using the sequences obtained in this 397 study and 98sequences from E. granulosus s.s. isolated from Algeria retrieved from GenBank. 398 1a: classification according to the host either human or animal; $1 \mathrm{~b}$ : classification according to 399 the geographical origin

400 Circles represent sequence type; the distance between circles represents the number of 401 mutations; circle diameter represents number of specimens with the corresponding sequence 402 type.

403 Accession numbers of sequences from GenBank used in this figure: AF408686.1, 404 KR349027.1, KR381826.1, KU925429.1, MG672128.1, MG672283.1, MG672284.1, 405 MG672285.1, MG672287.1, MG672288.1, MG672289.1, MG672291.1, MG672292.1, 406 MG672293.1, MG808283.1, MG808285.1, MG808287.1, MG808290.1, MG808292.1, 407 MG808293.1, MG808294.1, MG808295.1, MG808296.1, MG808298.1, MG808302.1, 408 MG808303.1, MG808307.1, MG808308.1, MG808309.1, MG808313.1, MG808315.1, 409 MG808316.1, MG808317.1, MG808318.1, MG808324.1, MG808327.1, MG808328.1, 410 MG808330.1, MG808331.1, MG808334.1, MG808336.1, MG808337.1, MG808338.1, 411 MG808339.1, MG808340.1, MG808343.1, MG808344.1, MG808345.1, MG808346.1, 412 MG808348.1, MG808349.1, KR349028.1, MG672290.1, MG808286.1, MG808305.1, 413 MG808321.1, MG808322.1, MG808347.1, KR349030.1, MG672286.1, MG808304.1, 414 MG808326.1, KT316341.1, MG808342.1, MG808341.1, KR349034.1, MG808335.1, 415 MG808329.1, MG808320.1, MG808297.1, MG808319.1, MG808310.1, MG808288.1, 416 MG808301.1, MG808284.1, MG808299.1, MG808291.1, MG808289.1, KR349029.1, 417 KR349033.1, MG808333.1, MG808314.1, MG808332.1, MG808325.1, MG808312.1, 418 MG808311.1, MG808306.1, MG808282.1, KR349032.1, KR349031.1, MG808300.1, 419 MG808323.1 
421 Fig. 2. Minimum spanning tree of the concatenated haplotypes (atp6, nd3, coxla, coxlb) from 422 this study (dark blue) and 12 sequences from E. granulosus s.s. isolated from Algeria 423 retrieved from GenBank (clear blue).

424 Accession numbers of sequences from GenBank used in this figure: MG672288 MG672291

425 MG672284 MG672290 MG672128 MG672283 MG672285 MG672286 MG672287

426 MG672289 MG672292 MG672293 MG682544

427 


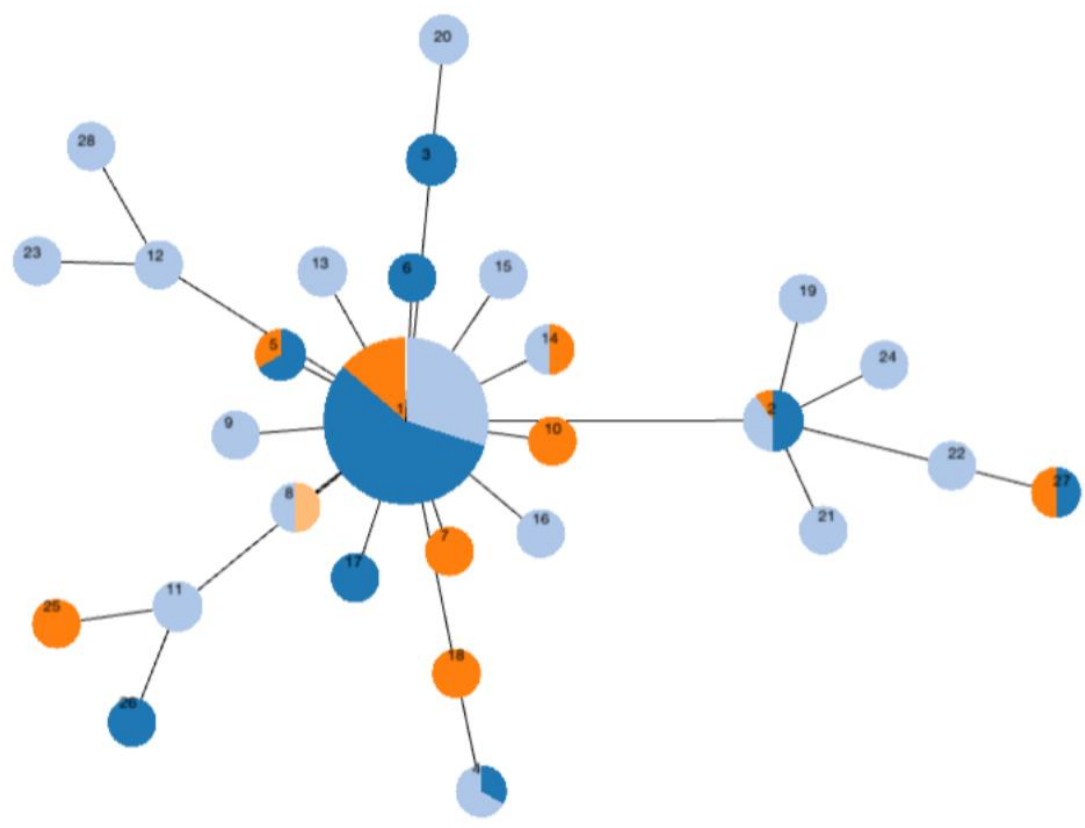

Homo sapien

Sheep

Cattle

Figure 1a

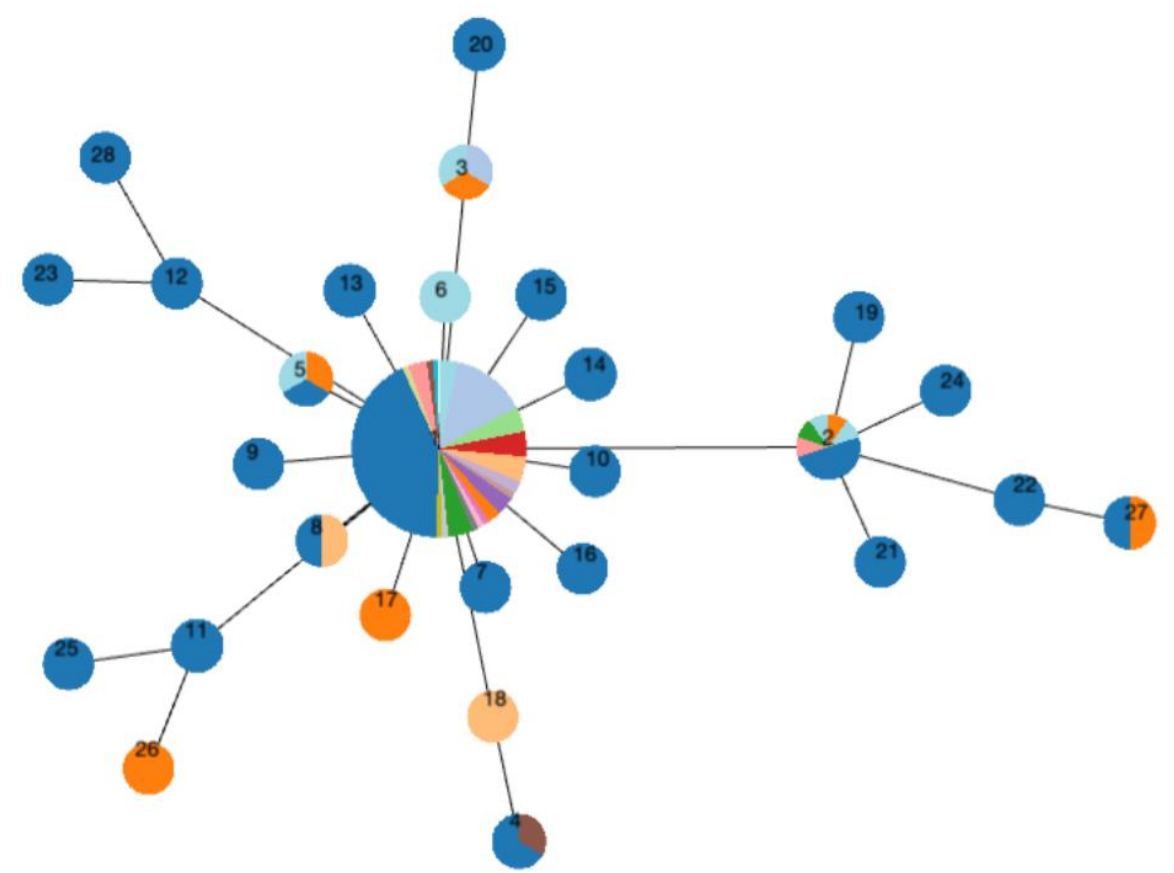

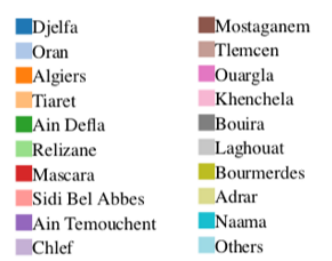

Figure $1 b$ 


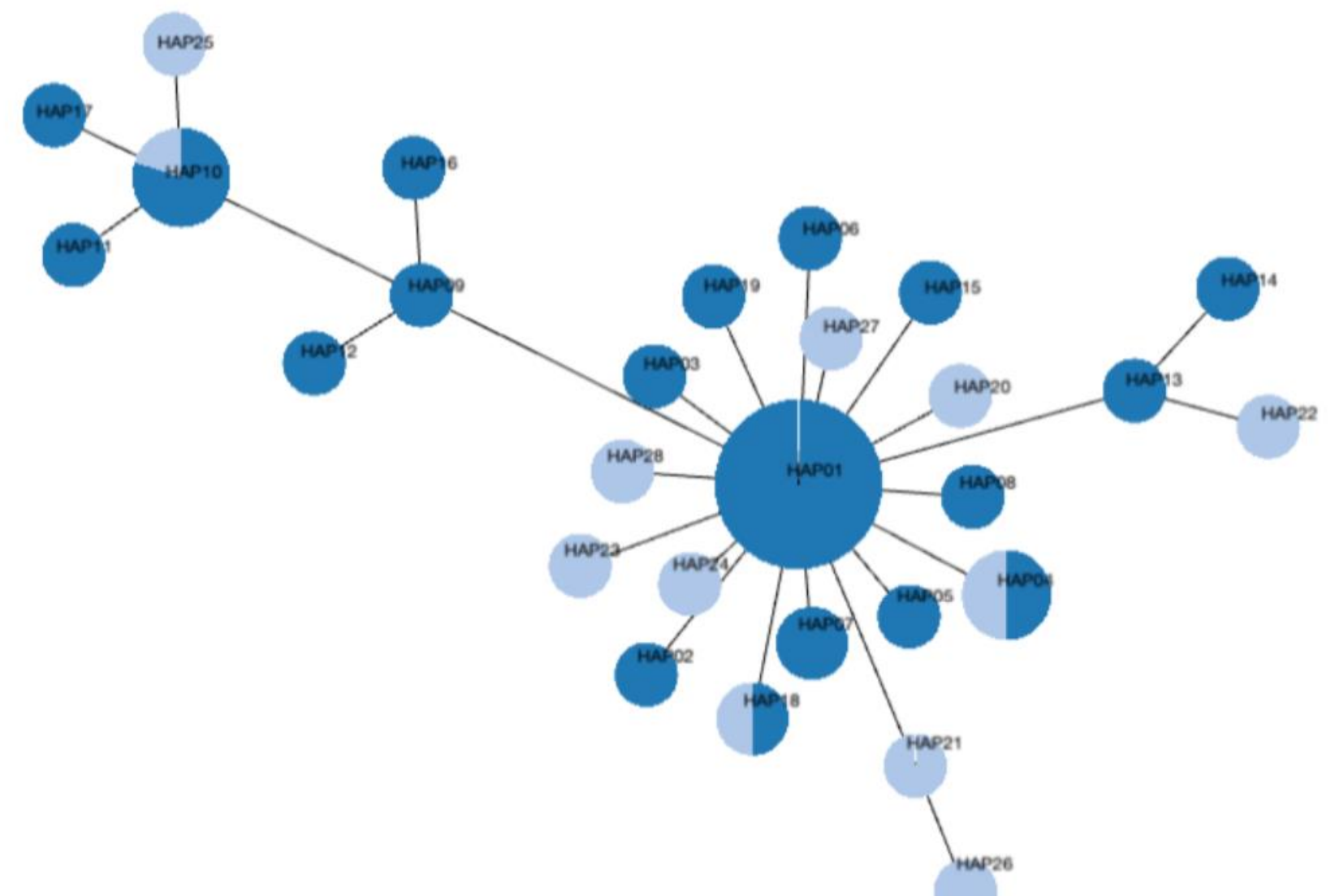

Figure 2

This study

Laatamna et al 2019 


\section{Supplementary data}

437 Table S1: Demographic characteristics of E. granulosus s.s. analyzed in this study

438 Table S2: Primers used for amplification and sequencing of E. granulosus s.l. mitochondrial 439 targets.

440

441 Figure S1: Algerian localities of origin of the E. granulosus s.s. tested in this study (red dots) 442 and from previous studies conducted in Algeria (Blue dots)

443 Figure S2: Genetic map showing the 4 loci used in this study and their respective position on 444 the mitochondrial genome of E. granulosus s.s. Nucleotide positions refer to the reference 445 genome Genbank accession number AB786664)

446

447 


\begin{tabular}{|c|c|c|c|}
\hline Isolates & Age & $\begin{array}{l}\text { Anatomic site } \\
\text { of isolation }\end{array}$ & Living place \\
\hline $\mathrm{F} 1$ & 41 & Liver & Oran \\
\hline $\mathrm{F} 2$ & 38 & Liver & Oran \\
\hline F3 & 35 & Liver & Oran \\
\hline $\mathrm{F} 4$ & 29 & Liver & Oran \\
\hline F6 & 60 & Liver & Oran \\
\hline F7 & 40 & Liver & Relizane \\
\hline F8 & 31 & Liver & Illizi \\
\hline F9 & 60 & Liver & Mascara \\
\hline F10 & 47 & Liver & Tiaret \\
\hline F11 & 27 & Liver & Chlef \\
\hline F12 & 22 & Liver & Tlemcen \\
\hline F13 & 68 & Liver & Ain Temouchent \\
\hline F14 & 25 & Liver & Oran \\
\hline F20 & 64 & Liver & Ain Temouchent \\
\hline $\mathrm{F} 21$ & 64 & Liver & Oran \\
\hline $\mathrm{F} 22$ & 43 & Liver & Mascara \\
\hline $\mathrm{F} 23$ & 54 & Liver & Oran \\
\hline F24 & 56 & Liver & Oran \\
\hline F25 & 32 & Liver & Relizane \\
\hline F26 & 29 & Liver & Tiaret \\
\hline F27 & 29 & Liver & Tiaret \\
\hline $\mathrm{F} 27^{1}$ & 29 & Liver & Tiaret \\
\hline F28 & 31 & Liver & Ain Temouchent \\
\hline F29 & 39 & Liver & Mostaganem \\
\hline F30 & 34 & Liver & Oran \\
\hline F31 & 60 & Liver & Oran \\
\hline $\mathrm{P} 1$ & 52 & Lungs & Mascara \\
\hline $\mathrm{P} 2$ & 32 & Lungs & Chlef \\
\hline $\mathrm{P} 3$ & 38 & Lungs & Adrar \\
\hline $\mathrm{P} 4$ & 35 & Lungs & Sidi Bel Abbes \\
\hline $\mathrm{P} 5$ & 59 & Lungs & Tiaret \\
\hline
\end{tabular}




\begin{tabular}{llll}
\hline P7 & 64 & Lungs & Mostaganem \\
\hline P8 & 37 & Lungs & Sidi Bel Abbes \\
\hline P10 & 16 & Lungs & Sidi Bel Abbes \\
\hline P11 & 20 & Lungs & Relizane \\
\hline P12 & 40 & Lungs & Ain Defla \\
\hline P14 & 63 & Lungs & Sidi Bel Abbes \\
\hline P15 & 31 & Lungs & Saida \\
\hline P16 & 19 & Lungs & El Bayadh \\
\hline P17 & 23 & Lungs & Tissemsilt \\
\hline P18 & 37 & Lungs & Oran \\
\hline P22 & 77 & Lungs & Mascara \\
\hline P23 & 21 & Lungs & Relizane \\
\hline P24 & 17 & Lungs & Naama \\
\hline
\end{tabular}

449 Table S1: Demographic characteristics of E. granulosus s.s. analyzed in this study

$450{ }^{1}$ Denote isolation from same patient

451 


\begin{tabular}{|c|c|c|c|c|c|}
\hline $\begin{array}{l}\text { Gene } \\
\text { target }\end{array}$ & Primers & Primers sequence & Position $^{1}$ & Fragment size & Previously described in \\
\hline \multirow[t]{2}{*}{ atp6 } & $\mathrm{F}$ & 5'-TCAATTTGAAGCGTTGGAGATAACTT & $5661-5686$ & 770 & Lavikainen et al., 2006 \\
\hline & $\mathrm{R}$ & 5'-GAAGGAACAATTGCCAACCC & $6411-6430$ & & Lavikainen et al., 2006 \\
\hline \multirow[t]{2}{*}{$n d 3$} & $\mathrm{~F}$ & 5'-TTGGGTATCCTTGGTCTCGT & $8407-8426$ & 550 & Lavikainen et al., 2006 \\
\hline & $\mathrm{R}$ & 5'-ATGACACAAAATTATTAGCAGTA & $8934-8956$ & & Lavikainen et al., 2006 \\
\hline \multirow[t]{2}{*}{ coxla } & $\mathrm{F}$ & 5'-TTTTTTGGGCATCCTGAGGTTTAT & $8878-8901$ & 444 & Bowles et al., 1992 \\
\hline & $\mathrm{R}$ & 5'-TAAAGAAAGAACATAATGAAAATG & $9327-9348$ & & Bowles et al., 1992 \\
\hline \multirow[t]{2}{*}{$\operatorname{cox} 1 b$} & $\mathrm{~F}$ & 5'-TTGTTGAATTGTTTAGTGGGTATG & $9762-9785$ & 471 & Lavikainen et al., 2006 \\
\hline & $\mathrm{R}$ & 5'-GCGGTAAATTCAAATCAGACAA & $10182-10205$ & & Lavikainen et al., 2006 \\
\hline
\end{tabular}

456

Table S2: Primers used for amplification and sequencing of E. granulosus s.l. mitochondrial targets.

458

$459{ }^{1}$ Position on the complete reference mitochondrial genome (Genbank accession number AB786664) 
This study

(1) Wilaya d'Adrar

(2) Wilaya d'Aïn Defla

(3) Wilaya d'Aïn Témouchent

(4) Wilaya de Chlef

(5) Wilaya d'El Bayadh

(6) Wilaya de Mascara

(7) Wilaya de Mostaganem

8 Wilaya de Naâma

(9) Wilaya d'Oran

(10) Wilaya de Relizane

(11) Wilaya de Saïda

(12) Wilaya de Sidi Bel Abbès

(13) Wilaya de Tiaret

(14) Wilaya de Tissemsilt

(15) Wilaya de Tlemcen

Previous studies

(1) Wilaya de Blida

(2) Wilaya de Bouira

(3) Wilaya de Bourmerdès

(4) Wilaya de Khenchela

(5) Wilaya de Laghouat

6 Wilaya d'Ouargla

(7) Wilaya de Tipaza

8 Wilaya de Tiaret

9 Wilaya d'Aïn Defla

(10) Alger

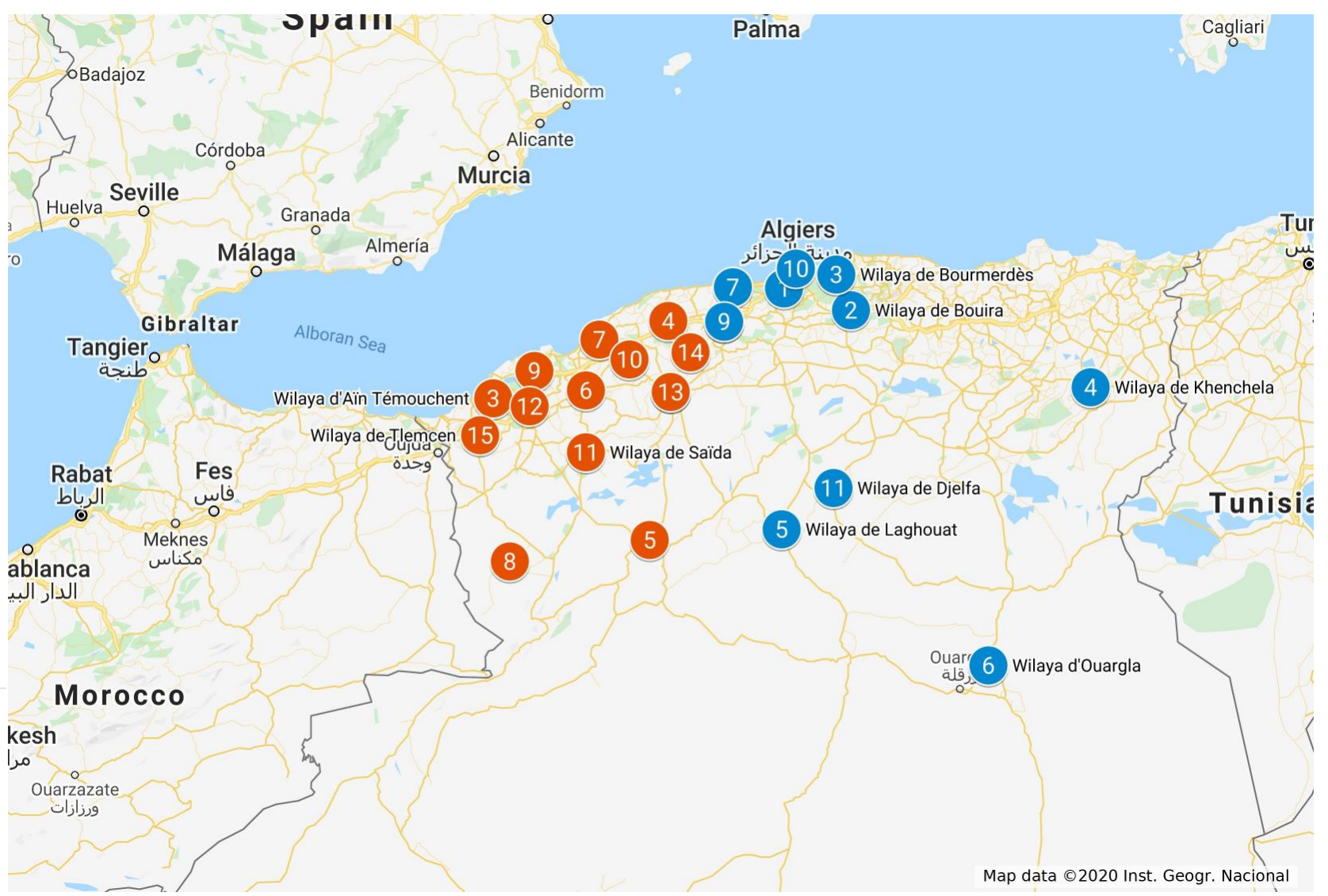

Figure S1: Algerian localities of origin of the E. granulosus strains tested in this study (red dots) and from previous studies conducted in Algeria (Blue dots) 
Figure S2

5767.6279

$8571 . .8918$

$9054 . .10727$

ATP6

NAD3

COX1

atp 6

$n d 3$

5661-6430

cox1a

8407-8956

8878-9348

9762-10205 